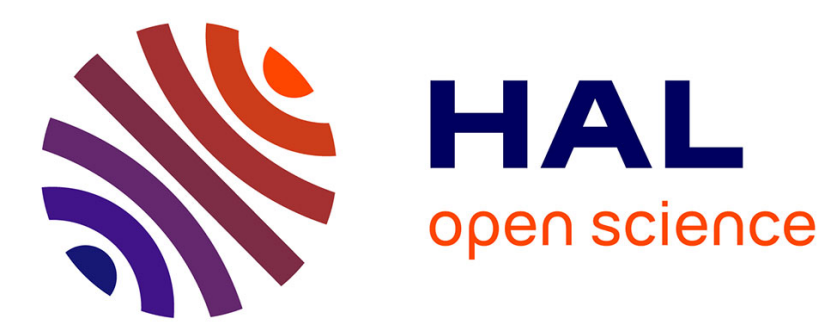

\title{
Blind Detection of Severely Blurred 1D Barcode
}

\author{
Noura Dridi, Yves Delignon, Wadih Sawaya, François Septier
}

\section{To cite this version:}

Noura Dridi, Yves Delignon, Wadih Sawaya, François Septier. Blind Detection of Severely Blurred 1D Barcode. Global Telecommunications Conference (GLOBECOM 2010), 2010 IEEE, Dec 2010, United States. pp.1-5. hal-00805761

\section{HAL Id: hal-00805761 https://hal.science/hal-00805761}

Submitted on 28 Mar 2013

HAL is a multi-disciplinary open access archive for the deposit and dissemination of scientific research documents, whether they are published or not. The documents may come from teaching and research institutions in France or abroad, or from public or private research centers.
L'archive ouverte pluridisciplinaire HAL, est destinée au dépôt et à la diffusion de documents scientifiques de niveau recherche, publiés ou non, émanant des établissements d'enseignement et de recherche français ou étrangers, des laboratoires publics ou privés. 


\title{
1D Barcode Blind Detection in Severe Environment
}

\author{
Noura Dridi, Yves Delignon, Wadih Sawaya and François Septier \\ Université Lille Nord de France, Institut TELECOM, TELECOM Lille1, LAGIS FRE 3303 CNRS \\ TELECOM Lille1, rue Guglielmo Marconi, BP 20145, 59653 Villeneuve d'Ascq, France \\ Email: Name@telecom-lille1.eu
}

\begin{abstract}
In this paper, we present a joint blind channel estimation and symbol detection for decoding a blurred and noisy $1 D$ barcode captured image. From an information transmission point of view, we show that the channel impulse response, the noise power and the symbols can be efficiently estimated by taking into account the signal structure such as the cyclostationary property of the hidden Markov process to estimate. Based on the Expectation-Maximisation method, we show that the new algorithm offers significative performance gain compared to classical ones pushing back the frontiers of the barcode technology.
\end{abstract}

\section{INTRODUCTION}

Barcode is an essential element of electronic data interchange. Since its birth in 1948, this technology is continuously growing up. Its success comes from the simplicity of the binary data representation and the efficiency of the reading device in a controlled acquisition environment. Originally, barcodes represent data through the width and the spacing of parallel lines, and may be referred to as 1D barcodes. Binary data can also be depicted in geometric patterns such as dots or squares within images known as 2D barcodes.

When shooted, the original barcode is degraded by blur caused by a bad focalisation and/or a camera movement, in addition to noise, the whole resulting in a blurred noisy received signal, Fig.1. In fact, two main types of blur can affect the received signal; the optical blur owing to bad focus and diffraction phenomena and the motion blur coming from the camera mobility with respect to the barcode. Many models have been proposed for these two kinds of blur such as parametric models [10], Gaussian models [4] and models based on the global transfer function [3] and [7].

In the scientific literature few works are related to 1D and 2D barcode coding. Pavlidis et al. [13] have published a study related to information theory fundamentals outlining the process for barcode design using error detection and correction techniques. Tsi et al. [14] have developed a method that allows the calculation of the working range in case of a CCDbased reader. Houni et al [8] have studied the performance in the framework of the information theory and they have proposed two new measures: the theoretical depth of field and the theoretical resolution. In [11], a method of reading linear barcodes using the Hough transform is proposed. The algorithm is designed to a particular type of barcode (Code39). Recently Ould Barikalla et al [12] have proposed a 2D barcode decoder using an iterative structure based on a factor graph representation. In all these works, decoders take into account the prior knowledge of the channel at the receiver. In practice, a training sequence enables the channel to be estimated but the estimation error leads to performance degradation of the decoder.

In this paper, we consider the decoding problem of barcode without training sequence. In particular, we propose a blind decoder that jointly estimates the channel and the symbols. This joint channel estimation and symbol detection can be performed, using probabilistic algorithm based on maximum likelihood criterion [1], [5], [6] and [9]. Here, we show that the channel state of a barcode is a cyclostationary process and we propose a new joint channel estimator and symbol detector based on the Expectation Maximisation algorithm which takes advantage of this property. By exploiting the cyclostationarity property, the number of paths of the channel state in the lattice diagram is reduced. As a consequence, the detector is more robust to noise and the detector performances are significantly improved.

The paper is organised as follow. In Section 2, both the barcode and the channel are modeled respectively, by a cyclostationary process and by a finite impulse response filter with an additive white Gaussian noise. Section 3 describes the Expectation Maximisation algorithm used for the channel estimation. Section 4 is focused on the symbol detection. Section 5 presents the results of a simulation study that has been conducted to assess the new algorithm's performance. Conclusions are given in Section 6.
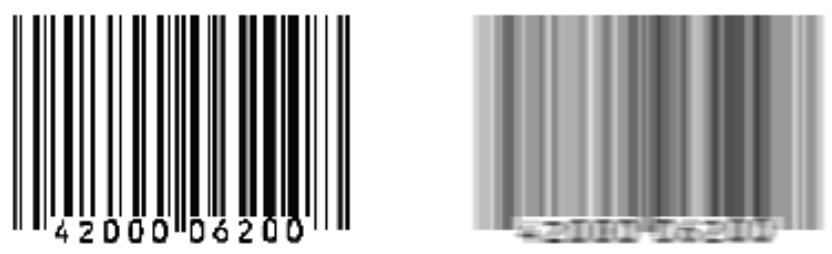

Fig. 1: Original and blurred barcode

\section{Notation}

$\lfloor x\rfloor$ returns the smallest integer value greater than or equal to $x . x(i: j)=\left(x_{i}, x_{i+1}, \ldots, x_{j}\right) . x^{T}$ is the classical matrix transpose.

\section{SYSTEM MODEL}

Let $x_{0}^{\prime}, x_{1}^{\prime} \ldots, x_{N-1}^{\prime}$ be a sequence of $N$ bits to transmit. Each bit is represented by a bar of width $r_{x}$. So the barcode is given by $x_{k}=x_{\left\lfloor\frac{k}{r_{x}}\right\rfloor}^{\prime}, k \in\{0, \ldots, K-1\}$ with $K=N r_{x}$. 
When shooted, the barcode is degraded by a blur coming from both the optical block and the movement of the camera which are modeled by a finite impulse response filter of length $L$. Besides, an additive white Gaussian noise takes into account the residual stochastic imperfections. Using matrix notation, the observed sequence $Y=\left(y_{0} \ldots y_{K-1}\right)^{T}$ is given by:

$$
y_{k}=H^{T} X_{k}+w_{k} \quad \forall k \in\{0, \ldots, K-1\}
$$

where $H=\left(h_{0} \ldots h_{L-1}\right)^{T}$ is the channel coefficients vector, $H$ is symmetric and decreasing from central maximum. Indeed, the blur is the result of the bad focalisation and the camera motion, which belongs to the class of this symmetric blur [3], [4], [10] and [7].

$X_{k}=\left(x_{k} \ldots x_{k-L+1}\right)^{T}$ is the displayed symbol vector and $w_{k}$ is an additive white Gaussien noise with variance $\sigma^{2} . X_{k}$ verifies the equation :

$$
X_{k}=B X_{k-1}+V_{k}
$$

With $B=\left(\begin{array}{cccc}0 & 0 & \cdots & 0 \\ & \ddots & & \vdots \\ \vdots & & \ddots & 0 \\ 0 & \cdots & 1 & 0\end{array}\right)$ and $V_{k}=\left(x_{k} 0 \ldots 0\right)^{T}$.

From (2), we deduce that $X_{k}$ is a Markovian process which belongs to a set $\left\{\xi^{0, r_{x}}, \ldots, \xi^{M-1, r_{x}}\right\}$ in which the cardinal number $M$ and the states are relied to both the channel length $L$ and the bar width $r_{x}$. Specifically, the number of states $M$ follows the equation :

$$
M= \begin{cases}2 L & \text { if } L \leq r_{x} \\ \sum_{i=0}^{r_{x}-1} 2^{\left(1+\left\lfloor(L-1) / r_{x}\right\rfloor\right)}-2\left(r_{x}-1\right) & \text { otherwise }\end{cases}
$$

Let's point out from equation (3) that $M$ is decreasing with $r_{x}$ which is an interesting property when dealing with the recovery of the information carried by the barcode. The distance between the various states becomes larger so that detection is less sensitive to the noise.

By denoting $A_{k}=\left(a_{m n}(k)\right)_{0 \leq m, n<M-1}$, the transition matrix of $X_{k}$ is given by :

$$
\begin{gathered}
a_{m n}(k)=P\left(X_{k}=\xi^{n, r_{x}} \mid X_{k-1}=\xi^{m, r_{x}}\right) \\
=\left\{\begin{array}{l}
\frac{1}{2} \delta\left(\xi^{n, r_{x}}(1: L-1)-\xi^{m, r_{x}}(0: L-2)\right) \text { if } \frac{k-1}{r_{x}} \in \mathbb{N} \\
\delta\left(\xi^{n, r_{x}}(1: L-1)-\xi^{m, r_{x}}(0: L-2)\right) \text { otherwise }
\end{array}\right.
\end{gathered}
$$

with $\delta($.$) the Kronecker symbol. As shown in equation (4),$ if $X_{k}$ is at the border of the bar, two transitions are possible, otherwise only one transition is allowed.

From equation (1) and the Markovian properties of $X=\left(X_{0} \ldots X_{K-1}\right)$, we deduce the distribution of $(X, Y)$ :

$$
P_{\theta}(Y, X)=P\left(X_{0}\right) \prod_{k=1}^{K-1} P\left(X_{k} \mid X_{k-1}\right) \prod_{k=0}^{K-1} f_{Y_{k} \mid X_{k}, \theta}\left(y_{k}\right)
$$

where $f_{Y_{k} \mid X_{k}, \theta}\left(y_{k}\right)=\frac{1}{\sqrt{2 \pi \sigma^{2}}} \exp -\frac{1}{2}\left(\frac{y_{k}-H^{T} X_{k}}{\sigma}\right)^{2}$, $P\left(X_{k} \mid X_{k-1}\right)$ is given by equation (4) and $\theta=\left(H, \sigma^{2}, r_{x}\right)^{T}$ is the parameter vector of the model.

From the described model, $X_{k}$ can be represented in a trellis diagram. Since the Markov process is cyclostationary, the lattice is cyclic so that the number of paths is reduced. As a consequence, the detection of the optimal sequence is simplified and more robust to noise.

\section{PARAMETER ESTIMATION}

\section{A. EM algorithm}

The maximum likelihood of $\theta$ is given by :

$$
\hat{\theta}=\arg \max _{\theta} f_{Y, \theta}(y)
$$

where $f_{Y, \theta}$ is the likelihood of the observation. Nevertheless in our application the likelihood maximisation is not tractable. On the other hand, when considering the complete data $(X, Y)$, the maximum likelihood estimation can be closely approximated by the Expectation-Maximisation algorithm (EM). This recursive algorithm consists of an iterative maximization of the auxilliary function $Q\left(\theta, \theta^{(i-1)}\right)$.

At the $i^{\text {eme }}$ iteration, the two following steps are performed: E step

$$
Q\left(\theta, \theta^{(i-1)}\right)=E_{X \mid Y, \theta^{(i-1)}}\left[\log \left(P_{\theta}(X, Y)\right)\right]
$$

M step

$$
\theta^{(i)}=\arg \max _{\theta} Q\left(\theta, \theta^{(i-1)}\right)
$$

In order to proceed to the estimation of the three components of $\theta, Q$ is first maximised with regards to $\left(H, \sigma^{2}\right)$ and the result is maximised relatively to the width $r_{x}$.

$$
\max _{\theta} Q\left(\theta, \theta^{(i-1)}\right)=\max _{r_{x}} \max _{H, \sigma^{2}} Q\left(\theta, \theta^{(i-1)}\right)
$$

When considering the special case where the width $r_{x}=1, X_{k}$ is a stationary Markov process and the estimation algorithm of $\left(H, \sigma^{2}\right)$ has been proposed by Kaleh et al. [9]. In general case, i.e. whatever the value of $r_{x}$, we show that the channel coefficients and the noise variance estimates are respectively given by these two equations:

$$
\begin{array}{r}
\left\{\sum_{k=0}^{K-1} \sum_{m=0}^{M-1} P_{\theta^{(i)}}\left(X_{k}=\xi^{m, r_{x}} \mid Y\right) \xi^{m, r_{x}^{T}} \xi^{m, r_{x}}\right\} H_{r_{x}}^{(i+1)} \\
=\sum_{k=0}^{K-1} \sum_{m=0}^{M-1} P_{\theta^{(i)}}\left(X_{k}=\xi^{m, r_{x}} \mid Y\right) y_{k} \xi^{m, r_{x}^{T}}
\end{array}
$$

and

$$
\begin{array}{r}
\sigma_{r_{x}}^{2^{(i+1)}}=\frac{1}{K} \sum_{k=0}^{K-1} \sum_{m=0}^{M} P_{\theta^{(i)}}\left(X_{k}=\xi^{m, r_{x}} \mid Y\right) \times \\
\left|y_{k}-H_{r_{x}}^{(i+1)} \xi^{m, r_{x}}\right|^{2}
\end{array}
$$

where $P_{\theta^{(i)}}\left(X_{k}=\xi^{m, r_{x}} \mid Y\right)$ is obtained by the forward backward algorithm [2].

We propose to consider the estimation of the width $r_{x}$ as well as the estimation of the channel impulse response. Moreover, the cyclostationarity of $X_{k}$ is exploited to optimise the accuracy of the estimates. 


\section{B. Joint estimation of $H$ and $\sigma^{2}$ given $r_{x}$}

For a given $r_{x}$, the estimates of $H_{r_{x}}$ and $\sigma_{r_{x}}^{2}$ are obtained by solving the equations (10) and (11), where the marginal posterior probability $P_{\theta^{(i)}}\left(X_{k}=\xi^{m, r_{x}} \mid Y\right)$ is recursively calculated by the forward backward algorithm adapted to the cyclostationarity of the hidden process $X_{k}$. When $r_{x}>1$, the algorithm chooses the optimal sequence in a reduced set of paths in the lattice diagram, since some transitions are not possible.

Let us note

$$
\gamma_{m}(k)=P_{\theta}\left(X_{k}=\xi^{m, r_{x}} \mid Y\right)
$$

$\forall m \in\{0, \cdots, M-1\} \forall k \in\{0, \cdots, K-1\}$, the marginal posterior distribution of $X_{k}$. One shows that :

$$
\gamma_{m}(k)=\frac{\alpha_{m}(k) \beta_{m}(k)}{\sum_{n=0}^{M-1} \alpha_{n}(k) \beta_{n}(k)}
$$

where $\alpha_{n}(k)$ and $\beta_{n}(k)$ are respectively the forward and the backward probabilities defined as follow :

$$
\begin{gathered}
\alpha_{m}(k)=P_{\theta}\left(Y_{1}=y_{0}, \ldots, Y_{k}=y_{k}, X_{k}=\xi^{m, r_{x}}\right) \\
\beta_{m}(k)=P_{\theta}\left(Y_{k+1}=y_{k+1}, \ldots, Y_{K-1}=y_{K-1} \mid X_{k}=\xi^{m, r_{x}}\right)
\end{gathered}
$$

These probabilities can be calculated recursively by :

$$
\begin{gathered}
\alpha_{m}(k+1)=\left[\sum_{n=0}^{M-1} \alpha_{n}(k) a_{n m}(k)\right] f_{m}\left(y_{k+1}\right) \\
\beta_{m}(k)=\sum_{n=0}^{M-1} \beta_{n}(k+1) a_{m n}(k) f_{n}\left(y_{k+1}\right)
\end{gathered}
$$

where

- $f_{n}\left(y_{k}\right)$ is the density probability function of $Y$ given $X_{k}=\xi^{n, r_{x}}$. From equation (1), the Gaussian density with mean $\xi^{n, r_{x}}$ and variance $\sigma^{2}$ is deduced.

- $a_{m n}(k)$ is the transition probability (4).

The initialisation step of the forward and the backward algorithm are:

$\alpha_{m}(1)=\pi_{m} f_{m}\left(y_{0}\right)$ and $\beta_{m}(K)=1, \forall m \in\{0, \cdots, M-1\}$

\section{Estimation of $r_{x}$}

$r_{x}$ is estimated using the maximum likelihood criterion

$$
\hat{r_{x}}=\arg \max _{r_{x}}\left(g\left(H_{r_{x}}, \sigma_{r_{x}}^{2}, r_{x}\right)\right)
$$

with $g$ is the pesudo likelihood

$$
g\left(H_{r_{x}}, \sigma_{r_{x}}^{2}, r_{x}\right)=\max _{H, \sigma^{2}} Q\left(\theta, \theta^{(i-1)}\right)
$$

$H_{r_{x}}$ and $\sigma_{r_{x}}^{2}$ are the estimates obtained using equations (10) and (11). Maximisation of $g$ with regard to $r_{x}$ is equivalent to maximisation of the likelihood. Since, explicit expression of the estimator $\hat{r_{x}}$ can not be identified, $g$ is calculated for all possible value of $r_{x} \in\{1, \ldots, K-1\}$. Next, we choose $\left(\hat{H}_{r_{x}}, \hat{\sigma}_{r_{x}}^{2}, \hat{r}_{x}\right)$ that gives the highest likelihood.

\section{Symbol DETECTION}

The optimal decision on symbol is obtained using the Marginalised Posterior Mode (MPM) criterion, which is based on the maximisation of the marginal posterior probability. For symbol $x_{k}^{\prime}$ from the transmitted sequence of length $N$, the decision is taken by:

$$
P_{\theta}\left(x_{k}^{\prime}=0 \mid Y\right) \underset{\hat{x}_{k}^{\prime}=0}{\stackrel{\hat{x}_{k}^{\prime}=1}{\lessgtr}} P_{\theta}\left(x_{k}^{\prime}=1 \mid Y\right)
$$

Let $\Omega^{j}$ be the set of realisations $\xi=\left(\xi^{0}, \ldots, \xi^{r_{x}-1}\right)$ of $X_{k r_{x}:(k+1) r_{x}-1}$ such as $x_{k}^{\prime}=j$. Since, there is a one to one relation between $x_{k}^{\prime}$ and $X_{k r_{x}:(k+1) r_{x}-1}$

$$
P_{\theta}\left(x_{k}^{\prime}=j \mid Y\right)=P_{\theta}\left(X_{k r_{x}:(k+1) r_{x}-1} \in \Omega^{j} \mid Y\right)
$$

With,

$$
\begin{array}{r}
P_{\theta}\left(X_{k r_{x}:(k+1) r_{x}-1} \in \Omega^{j} \mid Y\right)=\sum_{\xi \in \Omega^{j}} P_{\theta}\left(X_{k}=\xi^{0} \mid Y\right) \times \\
\prod_{l=1}^{l=r_{x}-1} P\left(X_{k+l}=\xi^{l+1} \mid X_{k+l-1}=\xi^{l}\right)
\end{array}
$$

Where, $P_{\theta}\left(X_{k}=\xi^{0} \mid Y\right)$ is obtained by the forward backward algorithm (III-B) and the transition probabilities are given by equation (4). This criterion takes into account the structure of the barcode signal, via the marginal probability and the transition probability matrix.

\section{JOINT CHANNEL AND RESOLUTION ESTIMATION ALGORITHM}

From, subsections (III-A), (III-B), (III-C) and (IV), we deduce the algorithm to joint channel and barcode resolution estimation and symbol detection (CRESD):

\footnotetext{
Algorithm 1 Joint channel and resolution estimation and symbol detection algorithm

1: for $r_{x}=1, \ldots, K-1$ do

2: Calculate the number of state and the transition probability matrix using equations (3) and (4).

3: Initialisation, $H^{(0)},\left(\sigma^{2}\right)^{(0)}$.

We denote by $\varepsilon$ a predetermined threshold

4: $\quad$ while $\left|H_{r_{x}}^{k}-H_{r_{x}}^{k+1}\right|>\varepsilon$ do

5: $\quad$ Calculate the marginal posterior probability using equations (16), (17) and (13).

Estimation of $H_{r_{x}}$ and $\sigma_{r_{x}}^{2}$ from (10) and (11). end while

8: $\quad$ Given $H_{r_{x}}$ and $\sigma_{r_{x}}^{2}$, calculate the pseudo likelihood using equation (19).

9: end for

10: Select the parameter $\hat{\theta}=\left\{\hat{H}_{r_{x}}, \hat{\sigma}_{r_{x}}^{2}, \hat{r_{x}}\right\}$ that maximises the pseudo likelihood (19).

11: Detection of symbol using equations (20), (21) and (22).
} 


\section{Simulations RESUlts}

In this section, the performance of the proposed algorithm is illustrated through numerical simulations.

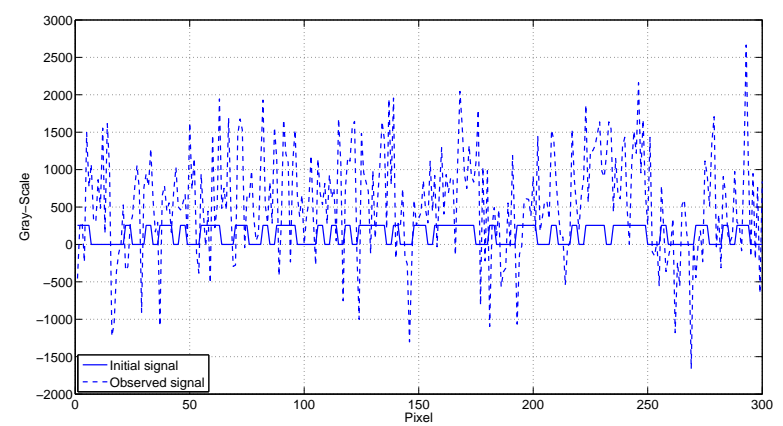

Fig. 2: Blurred signal with blur length $\mathrm{L}=10, r_{x}=3$ and $N=100$

Fig.2 shows the observed signal degraded by blur and noise distortion versus the initial transmitted signal. It confirms that the received signal can not be directly decoded. Indeed, a joint blur identification and symbol detection algorithm is required to efficiently recover the original signal.

In the following, a set of binary symbol $\{0,1\}$ have been considered and the results have been obtained with $N_{s}=1000$ Monte Carlo runs. For each run, a new sequence of length $N=100$ has been generated with a barcode resolution barcode $r_{x}=3$ and a new simulated channel of length $L=3$, the treshold $\varepsilon=10^{-5}$.

To analyse the performances of the proposed algorithm, we consider three cases:

- The Kaleh's algorithm [9], constructed without exploiting the cyclostationarity of the hidden Markov process.

- The CRESD algorithm, described in Algorithm1.

- A simple algorithm which does not take into account the channel model (1), but assumes known the resolution. The decision rule is:

$$
\left(\frac{1}{r_{x}} \sum_{i=k r_{x}}^{(k+1) r_{x}} y_{i}\right) \underset{\hat{x}_{k}^{\prime}=1}{\stackrel{\hat{x}_{k}^{\prime}=0}{\lessgtr}} \frac{1}{2}
$$

$\forall k \in\{0, \ldots, N-1\}$

In the experiments, $E_{b} / N_{0}$ is the energy per bit to noise power spectral density ratio, with energy per bit defined as:

$$
E_{b}=r_{x} H^{T} E\left[X_{k} X_{k}^{T}\right] H
$$

Estimation of the resolution $r_{x}$

Table (I) illustrates the performance of estimation of $r_{x}$. We calculate for different value of $r_{x}$ the error rate defined as $\rho=\frac{n_{b}}{N_{s}}$, where $n_{b}$ is the number of false detection and $N_{s}$ the number of trials. As shown in Table (I) the error rate decreases rapidly, with $E_{b} / N_{0}$. In fact, the error rate is due to noise distortion and interference intersymbol. The later, related

\begin{tabular}{|c|c|c|c|c|c|}
\hline$r_{x} \frac{E_{b}}{N_{0}}$ & $2 \mathrm{~dB}$ & $4 \mathrm{~dB}$ & $6 \mathrm{~dB}$ & $8 \mathrm{~dB}$ & $10 \mathrm{~dB}$ \\
\hline$r_{x}=2$ & 0.218 & 0.032 & 0.008 & 0.002 & 0.001 \\
\hline$r_{x}=3$ & 0.169 & 0.019 & 0.002 & 0.001 & 0 \\
\hline$r_{x}=4$ & 0.083 & 0.005 & 0 & 0 & 0 \\
\hline$r_{x}=5$ & 0.057 & 0.006 & 0 & 0 & 0 \\
\hline
\end{tabular}

TABLE I: $r_{x}$ error rate for channel length $L=3$ and block length $N=100$

to $r_{x} / L$, is reduced when $r_{x} / L>1$. Obviously, for the same value of $E_{b} / N_{0}$, estimation performance is better for $r_{x} / L>$ 1.

\section{Channel estimation}

The Root Mean Squared Error (RMSE), assessing the quality of the estimation of the channel, is defined as:

$$
R M S E=\sqrt{\frac{(H-\hat{H})^{T}(H-\hat{H})}{H^{T} H}}
$$

With $H$ and $\hat{H}$ are respectively the true channel coefficients vector and the estimated one.
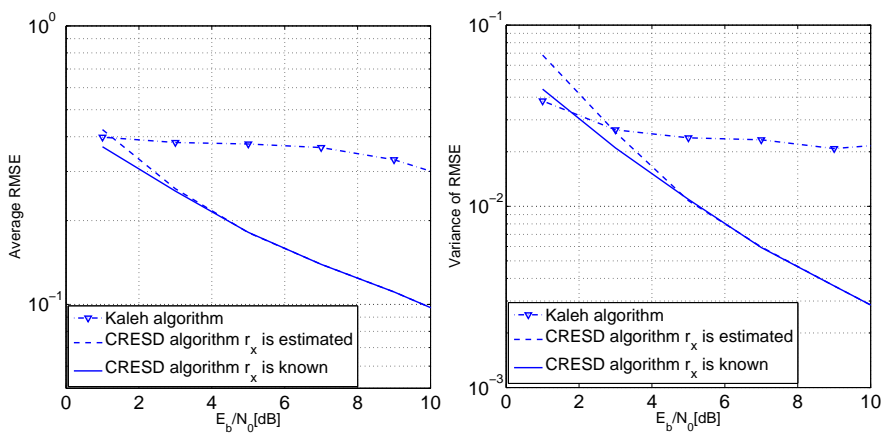

Fig. 3: RMSE vs $E_{b} / N_{0}$, for $\mathrm{N}=100, \mathrm{~L}=3$ and $r_{x}=3$

Fig. 3 shows the superiority of CRESD algorithm, in term of RMSE. This clearly illustrates the benefit of exploiting the cyclostationarity of the hidden Markov process in the trellis diagram. This leads to a more accurate estimation of the marginal posterior probability, which directly impacts the channel estimation equation(10). Besides, when the barcode resolution $r_{x}$ is estimated, the RMSE is larger for $E_{b} / N_{0}<$ $4 d B$, but becomes identical to the one when $r_{x}$ is known for $E_{b} / N_{0} \geq 4 d B$.

For the estimation of the noise variance, we note the same behavior. The $R M S E=6.7710^{-2}$ for the CRESD algorithm and it is equal to the double for the Kaleh's one $R M S E=$ $13.0510^{-2}$, for $E_{b} / N_{0}=5 d B$. Indeed, as channel coefficients, the estimation of the noise variance depends on the marginal posterior probability equation(11) which takes into account the true sequence structure of the barcode.

Now, the rapidity of convergence of the estimation is assessed. Fig. 4 shows that the CRESD is both more accurate and faster than the Kaleh's one. In this exemple,the convergence has 
been achieved at the $4^{\text {th }}$ iteration, while the Kaleh's algorithm requires twice as much iterations.

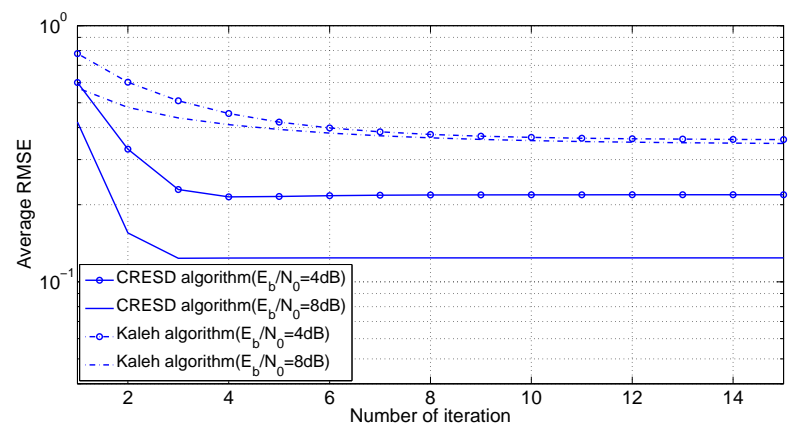

Fig. 4: Evolution of the RMSE with iterations, with $L=3$, $r_{x}=3$ and $N=100$

\section{Symbol detection}

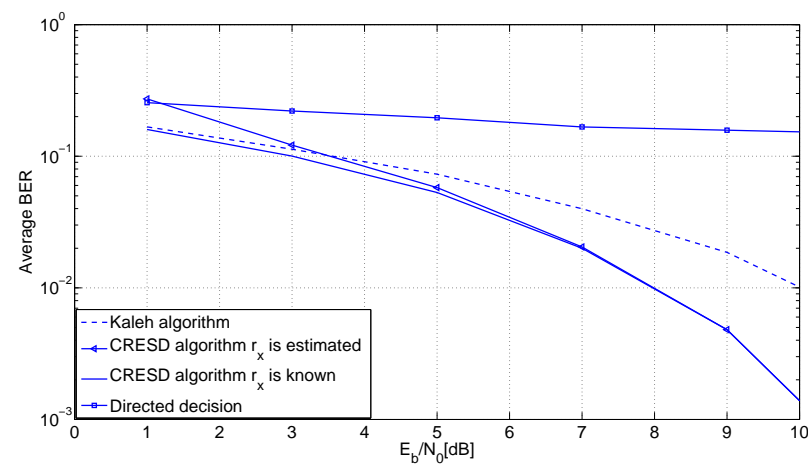

Fig. 5: BER performance for known and estimated $r_{x}, \mathrm{~L}=3$, $r_{x}=3, N=100$

Fig. 5 illustrates Bit Error Rate (BER) performance, curves with solid and dashed line correspond respectively to the CRESD algorithm and Kaleh's method. When $r_{x}$ is known, we note that BER performance is significantly improved using CRESD algorithm, and this performance gain increases with $E_{b} / N_{0}$. This result is explained by the fact that, with the CRESD algorithm, decision is taken in a trellis diagram constructed with respect of the original signal structure.

When $r_{x}$ is estimated, the resolution and channel estimation error lead to an increase of the BER for $E_{b} / N_{0}<4 d B$ for the CRESD algorithm. For the Kaleh's one, $r_{x}$ is supposed to be known. Nevertheless, the CRESD algorithm still performs better for $E_{b} / N_{0}>4 d B$, and the estimation of $r_{x}$ does not really degrades the overall performance. For $E_{b} / N_{0}>4 d B$, the curve obtained with and without estimation of $r_{x}$ are the same.

On the other hand, the poor performances of the method based on the decision without channel estimation confirms the need of a more accurate algorithm to ensure correct reconstruction of the original signal.

\section{CONCLUSION}

In this paper, we have proposed a blind channel estimation and symbol detection algorithm. Blur identification has been performed using the EM algorithm. Besides, given that the received signal is modeled as a hidden Markov chain, a version of the forward-backward algorithm, suitable to the cyclostationarity of the Markov process, has been proposed to estimate the marginal posterior probability. We have shown that, when the barcode structure is taken into account in the lattice, the estimation of the marginal posterior probability is easier and more accurate. This advantage directly impacts both the channel estimation and the symbol detection. Simulation results show a fast convergence, a large gain for the parameter estimation and a smaller symbol error rate compared to existing approaches. As a consequence, the CRESD algorithm can be efficiently used with larger intersymbol interference, thus enabling to read more compact barcode and to allow higher mobility between the barcode and the camera.

\section{REFERENCES}

[1] C. Anton-Haro and J. R. Fonollosa, "Blind channel estimation and data detection using hidden markov models," IEEE Trans. Signal Processing, vol. 45, pp. 241-247, 1997.

[2] L. Bahl, J. Cocke, F. Jelinek, and J. Raviv, "Optimal decoding of linear codes for minimizing symbol error rate (corresp)," IEEE Trans. Information. Theory, vol. 20, no. 2, pp. 284 - 287, Mar 1974.

[3] M. Cannon, "Blind deconvolution of spatially invariant image blurs with phase," IEEE Trans. Acoustics, Speech and Signal Processing, vol. 24 no. 1, pp. 58-63, Feb 1976.

[4] M. J. F. Chen, "An empirical identification method of gaussian blur parameter for image deblurring," IEEE Trans, Signal Processing, vol. 57, no. 7, pp. 2467-2478, July 2009.

[5] M. Feder and J. A. Catipovic, "Algorithms for joint channel estimation and data recovery-application to equalization in underwater communications," IEEE Journal, Oceanic Engineering, vol. 16, no. 1, pp. 42 -55, Jan 1991.

[6] J. A. R. Fonollosa and J. Vidal, "Application of hidden markov models to blind channel characterization and data detection," IEEE Int Conf Proceedings of the Acoustics, Speech, and Signal Processing, pp. 185188,1994

[7] D. B. Grennery, "Determination of optical transfer function by inspection of frequency-domain plot," Journal of The optical society of America, vol. 63, no. 12, pp. 1571-1577, Dec 1973.

[8] K. Houni, W. Sawaya, and Y. Delignon, "One-dimensional barcode reading : an information theoretic approach," Applied Optics, vol. 47, pp. 1025-1036, Mar 2008.

[9] G. K. Kaleh and R. Vallet, "Joint parameter estimation and symbol detection for linear or nonlinear unknown channels," IEEE Trans. Communications, vol. 42, no. 7, pp. 2406-2413, Jul 1994.

[10] M. E. Moghaddam, "A mathematical model to estimate out of focus blur," 5th Int Symp on Image and Signal Processing and Analysis, vol. 21, pp. 278-281, Sep 2007.

[11] R. Muniz, L. Junco, and A. Otero, "A robust software barcode reader using the hough transform," Int Conf on Information Intelligence and Systems Proceedings, pp. 313-319, 1999.

[12] M. Ouldbarikalla, W. Sawaya, Y. Delignon, and B. Pladeau, "New algorithm for 2d barcode detection," 11th Int Conf. Advanced. Communication. Technology, vol. 03, pp. 1979 -1981, Feb 2009.

[13] T. Pavlidis, J. Swartz, and Y. P. Wang, "Fundamentals of barcode information theory," Computer, vol. 23, no. 4, pp. 74-86, Apr 1990.

[14] D. Tsi, E. Marom, J. Katz, and J. Swartz, "System analysis of ccd-based bar code readers," Applied Optics, vol. 32, pp. 3504-3512, 1993. 